\title{
MICROSCALE ANALYSIS OF THE INFLUENCE OF VOID CONTENT, DISTRIBUTION AND SIZE ON FIBER-REINFORCED POLYMERS
}

\author{
$\underline{\text { O. Vallmajó }}^{1 *}$, A. Turon ${ }^{1}$, A. Arteiro ${ }^{2}$ \\ ${ }^{1}$ AMADE, Escola Politècnica Superior, Universitat de Girona, 17003 Girona, Spain \\ ${ }^{2}$ DEMec, Faculdade de Engenharia, Universidade do Porto, 4200-465 Porto, Portugal \\ *oriol.vallmajo@udg.edu
}

The increasing demand for Fiber-Reinforced Polymers (FRPs) for lightweight structures requires efficient manufacturing processes. However, predicting defect formation during production and the effect on the mechanical performance is still a matter of concern.

One of the main challenges for FRPs is the difficulty to predict their mechanical behavior due to the complex deformation and failure mechanisms, the presence of defects and the intrinsic variability of the material properties. At the microscale, the properties of the constituents, their spatial distribution and the defects arising from manufacturing play a critical role in the damage development. Thus, the experimental characterization of the damage onset and development is a very difficult and expensive task. However, accurate numerical simulations with advanced constitutive models can help understanding the mechanical behavior at the microscale (constituents level) and their translation to the mesoscale properties (ply level). To that end, a Representative Volume Element (RVE) of the composite material needs to be defined.

In this work, a computational micromechanical model is proposed and analyzed using a Finite Element (FE) software to determine the effect of defects, specifically voids, on the mechanical properties of FRPs. The fibers are randomly distributed in a micromechanical 3-D RVE in accordance to the fiber volume fraction, following the methods proposed in Refs. [1,2]. In the same way, the voids are distributed in the RVE according to the void content and their characteristic parameters. A parametric analysis is performed to analyze the effect of the main characteristics of the voids as described in Ref. [3], such as the spatial distribution, the void content and the void size, on the homogenized mechanical properties at the ply level. Finally, an uncertainty quantification and management analysis is employed to obtain material allowables on the mechanical properties at the mesoscale of the composite accounting for defects.

The results from the FE simulations help establishing the link between manufacturing defects, specifically the influence of voids, on the mechanical properties taking into account their associated variability. The proposed methodology is therefore a useful tool to determine the effect of defects on the mechanical response of FRPs.

\section{References}

[1] Melro, A.R., Camanho, P.P., \& Pinho, S.T. (2008). Generation of random distribution of fibres in long-fibre reinforced composites. Composites Science and Technology, 68(9), 2092-2102.

[2] Guerrero, J. M., Mayugo, J. A., Costa, J., \& Turon, A. (2018). A 3D Progressive Failure Model for predicting pseudo-ductility in hybrid unidirectional composite materials under fibre tensile loading. Composites Part A: Applied Science and Manufacturing, 107, 579-591.

[3] Zhang, D., Heider, D., \& Gillespie Jr, J. W. (2017). Determination of void statistics and statistical representative volume elements in carbon fiber-reinforced thermoplastic prepregs. Journal of Thermoplastic Composite Materials, 30(8), 1103-1119. 